\title{
Thermal and Voltage Side and Covert Channels and Attacks in Cloud FPGAs
}

\author{
Jakub Szefer \\ jakub.szefer@yale.edu \\ Yale University \\ New Haven, CT, USA
}

\begin{abstract}
Cloud FPGAs have been gaining interest in recent years due to the ability of users to request FPGA resources quickly, flexibly, and on-demand. In addition to the existing single-tenant deployments, where each user gets an access to a whole FPGA, recent academic proposals have looked at creating multi-tenant deployments, where multiple users share a single FPGA, e.g., [3]. In both settings, there is a large amount of infrastructure and physical resources that are shared among users. Sharing of the physical resources in data centers and processors is well known to lead to potential attacks, e.g., [4]. However, only recently have there been demonstrations of various security attacks that our group and others have shown to be possible in Cloud FPGA setting, e.g., [5].

This talk will discuss Cloud FPGA security from the perspective of side and covert channel attacks that arise due to these shared resources. It will first cover our recent work on thermal channels that can be used to create covert channels between users renting same FPGA over time [5]. These channels can create stealthy communication medium for leaking small amounts of sensitive information, e.g., cryptographic keys. As defense strategies, the talk will point out possible solutions at the system level and at the hardware level. At the system level, adding delays between when different users can access the same FPGA, or preventing users from being able to identify unique FPGA instances can mitigate the threats, but does increase overhead. At the hardware level, additional cooling to erase thermal information after users uses and FPGA, or new sensors to monitor FPGAs and generate an alert when excessive heat is detected are possible solutions that will be discussed.

The talk will also discuss recent work on voltage-based attacks that leverage custom circuits instantiated inside the FPGAs to measure voltage changes. Voltage-based channels can be used to leak sensitive information across FPGAs (in single-tenant or multitenant settings) [2], or can be combined with other existing attacks to perform cross-talk leakage inside the FPGAs (in multi-tenant settings) [1]. These attacks highlight the power of attacker when they are able to synthesize any circuit into a shared FPGA environment. Furthermore, even with certain restrictions on the types of designs that can be synthesized, this talk will show how attacks can be deployed. As defense strategies, the talk will point out possible new design check rules that can be used by Cloud FPGA providers.
\end{abstract}

Permission to make digital or hard copies of part or all of this work for personal or classroom use is granted without fee provided that copies are not made or distributed for profit or commercial advantage and that copies bear this notice and the full citation on the first page. Copyrights for third-party components of this work must be honored.

For all other uses, contact the owner/author(s).

FPGA '20, February 23-25, 2020, Seaside, CA, USA

(C) 2020 Copyright held by the owner/author(s)

ACM ISBN 978-1-4503-7099-8/20/02.

https://doi.org/10.1145/3373087.3375881
In light of the attacks and defenses, Cloud FPGA security remains a cat-and-mouse game. There is then the foremost need to better understand the existing and potential attacks - to design defenses and deploy them before malicious users try to launch such attacks. Only with proper understanding of the possible FPGA attacks, can secure Cloud FPGAs be created.

\section{CCS CONCEPTS}

- Security and privacy $\rightarrow$ Side-channel analysis and countermeasures; • Hardware $\rightarrow$ Reconfigurable logic and FPGAs;

\section{KEYWORDS}

FPGA security; Cloud computing; Side and covert channels

\section{SPEAKER'S BIO}

Jakub Szefer's research interests are at the intersection of computer architecture and hardware security. His research focuses on secure processor architectures, cloud security, hardware security and verification, physically unclonable functions, hardware FPGA implementation of cryptographic algorithms, and Cloud FPGA security. His research is supported through National Science Foundation and industry donations. He is an Assistant Professor of Electrical Engineering at Yale University, where he leads the Computer Architecture and Security Laboratory (CASLAB). Prior to joining Yale, he received Ph.D. and M.A. degrees in Electrical Engineering from Princeton University. He has received the NSF CAREER award in 2017. Most recently, Jakub is the author of a new book: "Principles of Secure Processor Architecture Design", published in 2018; and he has been promoted to the IEEE Senior Member rank in 2019.

\section{ACKNOWLEDGMENTS}

This work was supported in part by National Science Foundation's grant number 1901901.

\section{REFERENCES}

[1] Ilias Giechaskiel, Ken Eguro, and Kasper Bonne Rasmussen. 2019. Leakier Wires: Exploiting FPGA Long Wires for Covert- and Side-Channel Attacks. ACM Transactions on Reconfigurable Technology and Systems (TRETS) 12, 3 (Sept. 2019), 1-29.

[2] Ilias Giechaskiel, Kasper Rasmussen, and Jakub Szefer. 2020. CAPSULe: CrossFPGA Covert-Channel Attacks through Power Supply Unit Leakage. In Proceedings of the IEEE Symposium on Security and Privacy (S\&P).

[3] Ahmed Khawaja, Joshua Landgraf, Rohith Prakash, Michael Wei, Eric Schkufza, and Christopher J. Rossbach. 2018. Sharing, Protection, and Compatibility for Reconfigurable Fabric with AmorphOS. In Proceedings of the USENIX Symposium on Operating Systems Design and Implementation (OSDI).

[4] Jakub Szefer. 2019. Survey of Microarchitectural Side and Covert Channels, Attacks, and Defenses. Fournal of Hardware and Systems Security 3, 3 (Sept. 2019), 219-234.

[5] Shanquan Tian and Jakub Szefer. 2019. Temporal Thermal Covert Channels in Cloud FPGAs. In Proceedings of the International Symposium on Field-Programmable Gate Arrays (FPGA). 